\title{
Final results from the OPERA experiment in the CNGS neutrino beam
}

\author{
Matteo Tenti* on behalf of the OPERA Collaboration \\ INFN Sezione di Bologna, I-40127 Bologna, Italy \\ E-mail: tenti@bo.infn.it
}

\begin{abstract}
The OPERA experiment at the Gran Sasso Laboratory was designed to study $v_{\mu} \rightarrow v_{\tau}$ oscillations in appearance mode in the CERN-to-Gran Sasso neutrino beam. We report on the final analysis of the full data sample based on looser selection criteria than in previous analyses and multivariate approach. Oscillation parameters have been determined with a reduced statistical uncertainty, and the discovery of tau neutrino appearance is confirmed with an improved significance level. Moreover, the search for electron neutrino events has been extended to the full dataset exploiting an improved method for the electron neutrino energy estimation. New limits have been set in the framework of the $3+1$ neutrino model.
\end{abstract}

The 20th International Workshop on Neutrinos (NuFact2018)

12-18 August 2018

Blacksburg, Virginia

\footnotetext{
* Speaker.
} 


\section{The OPERA experiment}

The OPERA experiment [1] was designed to study $v_{\mu} \rightarrow v_{\tau}$ oscillations in appearance mode. The OPERA detector, located at the underground Gran Sasso Laboratory (LNGS), was exposed to the CERN to Gran Sasso (CNGS) muon neutrino beam [2, 3] produced at a distance of $730 \mathrm{~km}$. The average neutrino energy was about $17 \mathrm{GeV}$, the $\bar{v}_{\mu}$ fraction was $2.1 \%$ in terms of expected CC interactions, the sum of $v_{e}$ and $\bar{v}_{e}$ was below $1 \%$, while the prompt $v_{\tau}$ contamination was negligible.

The detector was a hybrid apparatus made of an emulsion and lead target with a total mass of about $1.25 \mathrm{kt}$, complemented by electronic detectors. The general structure consisted of two identical supermodules (SM). Each SM was made of a target section, composed of 31 target walls, and a muon spectrometer. Each target wall was an assembly of horizontal trays loaded with target units, hereafter called bricks. Each brick consisted of 57 emulsion films, $300 \mu \mathrm{m}$ thick, interleaved with 56 lead plates, $1 \mathrm{~mm}$ thick, with a $12.7 \times 10.2 \mathrm{~cm}^{2}$ cross section, a thickness of $7.5 \mathrm{~cm}$ corresponding to about ten radiation lengths and a mass of $8.3 \mathrm{~kg}$. Downstream of each target wall, two orthogonal planes of scintillator strips (target tracker detector) recorded the position and the energy deposition of charged particles [4]. Finally, a magnetic spectrometer instrumented with resistive plate chambers and high-resolution drift tubes was used to identify muons and measure their charge and momentum [1]. A detailed description of the OPERA detector can be found in [1].

\section{Tau neutrino appearance}

The appearance of the $v_{\tau}$ was identified by the detection of characteristic decay topologies of the tau lepton produced in the CC interactions. Kinematical selection criteria were applied to reduce the background coming from the processes that mimic the $\tau$ decay topologies, which are (i) the decays of charmed particles produced in $v_{\mu} \mathrm{CC}$ interactions; (ii) reinteractions of hadrons from $v_{\mu}$ events occurring in lead plates; (iii) the large-angle scattering of muons produced in $v_{\mu}$ $\mathrm{CC}$ interactions. A sample corresponding to $17.97 \times 10^{19}$ protons on target has been collected from 2008 to 2012 and resulted in 19505 neutrino interactions in the target fiducial volume. In 2015, five $v_{\tau}$ candidates were reported with an expected background of $0.25 \pm 0.05$ events. Candidates were selected by applying defined cuts on kinematical and topological parameters. The discovery of $v_{\mu} \rightarrow v_{\tau}$ oscillations was assessed with a significance of 5.1 $\sigma$ [6].

In 2018 a new analysis based on a multivariate approach for event identification, fully exploiting the expected features of $v_{\tau}$ events was performed [7]. It was applied on candidate events preselected with looser cuts than those applied in the previous cut-based approach. Ten tau neutrino candidates were identified in the full OPERA dataset with an expected number of background events of $2.0 \pm 0.4$. The significance of the discovery increased to $6.1 \sigma$.

Assuming maximal mixing and $v_{\tau} \mathrm{CC}$ interaction cross section as in default implementation provided by GENIE v2.6 [8, 9], an interval of $\left|\Delta m_{32}^{2}\right|=\left(2.7_{-0.6}^{+0.7}\right) \times 10^{-3} \mathrm{eV}^{2}$ was derived at $68 \%$ C.L. using the Feldman-Cousins method [10]. This is the first result obtained in appearance mode and it is consistent with the disappearance results from different experiments, including the global best-fit value [11]. Alternatively, by fixing $\left|\Delta m_{32}^{2}\right|$ at the global best-fit value $\left(2.50 \times 10^{3} \mathrm{eV}^{2}\right)$ and 
maximal mixing $\sin ^{2} 2 \theta_{23}=1$, the $v_{\tau} \mathrm{CC}$ cross section on the lead target can be estimated [12]. The total flux integrated cross section was estimated to be $\langle\sigma\rangle_{\text {meas }}=\left(5.1_{-2.0}^{+2.4}\right) \times 10^{-36} \mathrm{~cm}^{2}$.

\section{Electron neutrino search}

The OPERA nuclear emulsion granularity allowed the reconstruction of electromagnetic showers and thus the identification of $v_{e} \mathrm{CC}$ interactions. A dedicated procedure [14], balancing computation time and detection efficiency, was defined to systematically search for $v_{e}$ events. A total of 35 $v_{e}$ events were observed. The number is compatible with the expected $v_{e}$ from the beam contamination (30.7 \pm 3.2$)$, together with two main sources of background: $\pi^{0}$ 's misidentified electrons in neutrino interactions without a reconstructed muon $(0.5 \pm 0.5)$ events and $v_{\tau} \mathrm{CC}$ interactions with $\tau$ decaying into an electron $(0.7 \pm 0.2)$ events.

Using the global best-fit values [11] for $\theta_{13}, \theta_{23}$ and $\Delta \mathrm{m}_{\mathrm{atm}}^{2}$, assuming $\delta_{\mathrm{CP}}=0$ and neglecting matter effects, a total of $34.3 \pm 3.5 v_{e}$ candidate events were expected in the whole energy range. The number of observed events is compatible with the three flavors oscillation model and an upper limits on $\sin ^{2} 2 \theta_{13}<0.43$ was set at $90 \%$ C.L. in the optimized neutrino energy range $[0-40] \mathrm{GeV}$.

\section{Constraints on $3+1$ model}

Some experimental results $[15,16,17,18]$ may hint to oscillations involving additional sterile neutrinos with a mass of $\mathrm{O}\left(1 \mathrm{eV}^{2}\right)$. In presence of a fourth sterile neutrino $(3+1$ model), the oscillation probability is a function of a $4 \times 4$ mixing matrix $U$ and of three squared mass differences. OPERA can exploit jointly tau and electron neutrino observed events to constrain combinations of the sterile neutrino parameters. The predictions of the $3+1$ model was evaluated using the GLoBES software [19, 20]. The parameter $\Delta \mathrm{m}_{21}^{2}$ was fixed to the gobal best-fit value [11], a Gaussian external constraint on $\Delta \mathrm{m}_{31}^{2}$ was set using mean and sigma from a global fit [11], while only positive values of $\Delta \mathrm{m}_{41}^{2}$ were considered since negative values are disfavoured by results on the sum of neutrino masses from cosmological surveys [22]. Matter effects were taken into account with the Earth density approximated by a constant value estimated with the PREM [20, 21] onion shell model.

The systematic uncertainty on the expected number of tau neutrino candidates is largely dominated by the limited knowledge of $v_{\tau}$ interaction cross section and the detection efficiency. It is conservatively set to $20 \%$. The systematic error on the expected number of electron neutrino candidates, due to beam normalization and efficiency uncertainties is $10 \%(20 \%)$ for events with neutrino energy larger (smaller) than $10 \mathrm{GeV}$.

The profile likelihood ratio was used as test statistic and the Wilks' theorem was used to extract a preliminary exclusion regions on $\sin ^{2} 2 \theta_{\mu \tau}=4\left|U_{\mu 4}\right|^{2}\left|U_{\tau 4}\right|^{2}$ and $\Delta \mathrm{m}_{41}^{2}$ plane (Fig. 1a) comparing the visible energy distribution of the observed $v_{\tau}$ events with the expected one. For $\Delta \mathrm{m}_{41}^{2} \gtrsim 1 \mathrm{eV}^{2}$, the $90 \%$ C.L. upper limit on $\sin ^{2} 2 \theta_{\mu \tau}$ is 0.099 . Furthermore an exclusion regions on $\sin ^{2} 2 \theta_{\mu e}=4\left|U_{\mu 4}\right|^{2}\left|U_{e 4}\right|^{2}$ and $\Delta \mathrm{m}_{41}^{2}$ plane (Fig. 1b) was preliminarily evaluated comparing the reconstructed energy distribution of the observed $v_{e}$ events with the expected one. At large $\left|\Delta \mathrm{m}_{41}^{2}\right|$ values, the $90 \%$ C.L. upper limit on $\sin ^{2} 2 \theta_{\mu e}$ is 0.019 . 


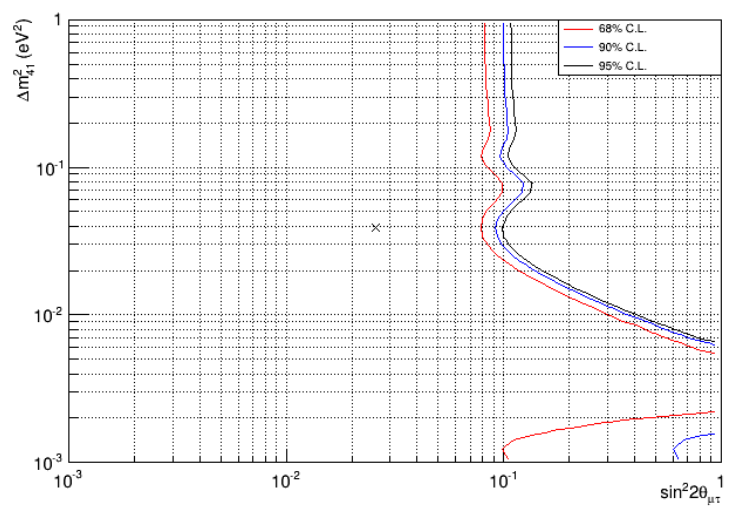

(a)

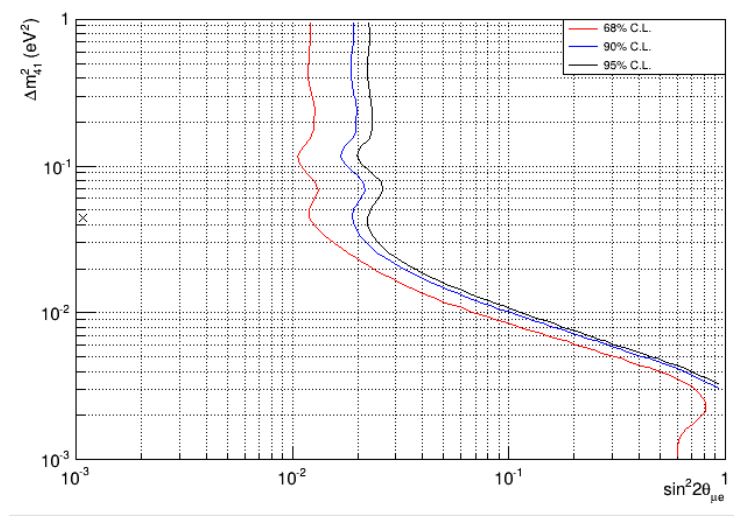

(b)

Figure 1: Preliminary exclusion regions on $\sin ^{2} 2 \theta_{\mu \tau}=4\left|U_{\mu 4}\right|^{2}\left|U_{\tau 4}\right|^{2}$ and $\Delta \mathrm{m}_{41}^{2}$ plane (a) and on $\sin ^{2} 2 \theta_{\mu e}=4\left|U_{\mu 4}\right|^{2}\left|U_{e 4}\right|^{2}$ and $\Delta \mathrm{m}_{41}^{2}$ plane (b) at $68 \%$ (red), $90 \%$ (blue) and $95 \%$ (black) C.L.

\section{Conclusions}

The OPERA experiment was taking data from 2008 to 2012. Five $v_{\tau}$ candidates were observed applying a selection on specific kinematical and topological parameters. The non-null observation of $v_{\mu} \rightarrow v_{\tau}$ oscillations was excluded with a significance greater than $5 \sigma$. In a subsequent analysis based on looser selection criteria, a sample of ten events was collected. Using a multivariate approach the significance of the observation increased to $6.1 \sigma$ and the $\Delta \mathrm{m}_{32}^{2}$ measurement was improved. Moreover a systematic search for $v_{e}$ events lead to a number of observed $v_{e}$ interactions compatible with the expectations in the three flavors neutrino model. Finally, the $v_{e}$ and $v_{\tau}$ samples were jointly used to derive limits on oscillation parameters in the $3+1$ neutrino model.

\section{References}

[1] R. Acquafredda et al., The OPERA experiment in the CERN to Gran Sasso neutrino beam, JINST 4 , P04018 (2009).

[2] G. Acquistapace et al., The CERN neutrino beam to Gran Sasso (NGS), CERN-98-02, INFN/AE-98/05.

[3] R. Baldy et al., The CERN Neutrino beam to Gran Sasso (NGS), CERN-SL-99-034-DI, CERN-SL-99-34-DI, INFN-AE-99-05, INFN-AE-99-5.

[4] T. Adam et al., The OPERA experiment target tracker, Nucl. Instrum. Meth. A 577 (2007) 523 [physics/0701153].

[5] A. Anokhina et al. [OPERA Collaboration], Emulsion sheet doublets as interface trackers for the OPERA experiment, JINST 3 (2008) P07005 [arXiv: 0804.1985 [physics.ins-det] ].

[6] N. Agafonova et al. [OPERA Collaboration], Discovery of $\tau$ Neutrino Appearance in the CNGS Neutrino Beam with the OPERA Experiment, Phys. Rev. Lett. 115 (2015) no.12, 121802 [arXiv:1507.01417 [hep-ex]]. 
[7] N. Agafonova et al. [OPERA Collaboration], Final Results of the OPERA Experiment on $v_{\tau}$ Appearance in the CNGS Neutrino Beam, Phys. Rev. Lett. 120 (2018) no.21, 211801 Erratum: [Phys. Rev. Lett. 121 (2018) no.13, 139901] [arXiv:1804.04912 [hep-ex]].

[8] C. Andreopoulos et al., The GENIE Neutrino Monte Carlo Generator, Nucl. Instrum. Meth. A 614 (2010) 87 [arXiv:0905.2517 [hep-ph]].

[9] C. Andreopoulos, C. Barry, S. Dytman, H. Gallagher, T. Golan, R. Hatcher, G. Perdue and J. Yarba, The GENIE Neutrino Monte Carlo Generator: Physics and User Manual, arXiv: 1510.05494 [hep-ph].

[10] G. J. Feldman and R. D. Cousins, A Unified approach to the classical statistical analysis of small signals, Phys. Rev. D 57 (1998) 3873 [physics/9711021 [physics.data-an]].

[11] M. Tanabashi et al. [Particle Data Group], Review of Particle Physics, Phys. Rev. D 98 (2018) no.3, 030001.

[12] A. Anokhina et al. [OPERA Collaboration], Study of the effects induced by lead on the emulsion films of the OPERA experiment, JINST 3 (2008) P07002 [arXiv: 0805.0123 [physics.ins-det]].

[13] T. Katori and M. Martini, Neutrino-nucleus cross sections for oscillation experiments, J. Phys. G 45 (2018) no.1, $013001[\operatorname{arXiv}: 1611.07770$ [hep-ph]].

[14] N. Agafonova et al. [OPERA Collaboration], Search for $v_{\mu} \rightarrow v_{e}$ oscillations with the OPERA experiment in the CNGS beam, JHEP 1307 (2013) 004 Addendum: [JHEP 1307 (2013) 085] [arXiv:1303.3953 [hep-ex]].

[15] C. Giunti and M. Laveder, Statistical Significance of the Gallium Anomaly, Phys. Rev. C 83 (2011) 065504 [arXiv: 1006.3244 [hep-ph] ].

[16] G. Mention, M. Fechner, T. Lasserre, T. A. Mueller, D. Lhuillier, M. Cribier and A. Letourneau, The Reactor Antineutrino Anomaly, Phys. Rev. D 83 (2011) 073006 [arXiv: 1101.2755 [hep-ex]].

[17] A. A. Aguilar-Arevalo et al. [MiniBooNE Collaboration], Improved Search for $\bar{v}_{\mu} \rightarrow \bar{v}_{e}$ Oscillations in the MiniBooNE Experiment, Phys. Rev. Lett. 110 (2013) 161801 [arXiv:1303.2588 [hep-ex]].

[18] A. Aguilar-Arevalo et al. [LSND Collaboration], Evidence for neutrino oscillations from the observation of anti-neutrino(electron) appearance in a anti-neutrino(muon) beaml, Phys. Rev. D 64 (2001) 112007 [hep-ex/0104049].

[19] P. Huber, M. Lindner and W. Winter, Simulation of long-baseline neutrino oscillation experiments with GLoBES (General Long Baseline Experiment Simulator), Comput. Phys. Commun. 167 (2005) 195 [hep-ph/ 0407333$].$

[20] P. Huber, J. Kopp, M. Lindner, M. Rolinec and W. Winter, New features in the simulation of neutrino oscillation experiments with GLoBES 3.0: General Long Baseline Experiment Simulator, Comput. Phys. Commun. 177 (2007) 432 [hep-ph/ 0701187 ].

[21] A. M. Dziewonski and D. L. Anderson, Preliminary reference earth model, Phys. Earth Planet. Interiors 25 (1981) 297.

[22] P. A. R. Ade et al. [Planck Collaboration], Planck 2015 results. XIII. Cosmological parameters, Astron. Astrophys. 594 (2016) A13 [arXiv:1502.01589 [astro-ph. CO]]. 
[23] P. Astier et al. [NOMAD Collaboration], Final NOMAD results on muon-neutrino $\rightarrow$ tau-neutrino and electron-neutrino $\rightarrow$ tau-neutrino oscillations including a new search for tau-neutrino appearance using hadronic tau decays, Nucl. Phys. B 611 (2001) 3 [hep-ex/0106102].

[24] E. Eskut et al. [CHORUS Collaboration], Final results on $n u(m u) \rightarrow n u(t a u)$ oscillation from the CHORUS experiment, Nucl. Phys. B 793 (2008) 326 [arXiv: 0710.3361 [hep-ex]].

[25] B. Armbruster et al. [KARMEN Collaboration], Upper limits for neutrino oscillations muon-anti-neutrino $\rightarrow$ electron-anti-neutrino from muon decay at rest, Phys. Rev. D 65 (2002) 112001 [hep-ex/0203021]. 